\title{
Hubble Space Telescope and Ground-Based Optical Imaging of the Proto-Planetary Nebula M1-92
}

\author{
Susan R. Trammell and Robert W. Goodrich \\ University of Chicago; W. M. Keck Observatory \\ Background
}

We present HST and ground-based images of the bipolar proto-planetary nebula M192. Trammell et al. (1993) studied M1-92 using spectropolarimetry and concluded that the continuum emission is reflected from the inner regions of the object, the forbidden lines are formed locally in the bipolar lobes, and the permitted lines are partially reflected and partially formed in the bipolar lobes. The emission produced in the lobes of M1-92 is indicative of shock heating, $\mathrm{V}_{s}=60-100 \mathrm{~km} \mathrm{~s}^{-1}$. Shock emission dominates the optical line spectra of the lobes of M1-92 and is a powerful probe into the properties of the mass loss occurring between the AGB and PN phases of evolution.

We obtained ground-based images of M1-92 through filters isolating the emission lines of $\mathrm{H} \alpha$, [S II] $\lambda \lambda 6717,31,[\mathrm{OI}] \lambda 6300$, and an adjacent continuum band with the McDonald Observatory $2.1 \mathrm{~m}$ telescope. In these ground-based images the [S II] and [O I] distributions are concentrated in diffuse knots in the bipolar lobes. This was previously noted by Solf (1994) for the [S II].

\section{HST Images}

In order to investigate the detailed morphology of M1-92, we obtained WFPC2 images of this object on 24 April 1996. We used four narrow-band filters with passbands similar to the filters used for the ground-based observations. The [O I] and [S II] images isolate shock emission produced in the lobes of M1-92. The continuum emission traces light that is reflected into our line of sight by dust in the bipolar lobes and the $\mathrm{H} \alpha$ emission is a combination of reflected and locally emitted emission.

The morphology is similar in the $\mathrm{H} \alpha$ and continuum images (Figure 1.1). The central star is clearly visible between the lobes. The central star was not easily separated from the bright lobe in the ground-based images. The overall distribution of continuum emission is smooth across the lobes. There is no evidence for a clumpy distribution of material or ring or arc-like structure that would indicate episodic mass loss.

The [O I] and [S II] emission is concentrated in compact regions in the bright lobe and a series of clumps in the fainter lobe. The shock emission is the result of a collimated outflow impacting the bipolar lobes. We estimate the opening angle of the jet to be 10 -20 degrees based on the spot size, a distance of $4.5 \mathrm{kpc}$, and an inclination of the system of 55 degrees. The jet appears to be misaligned with the bipolar axis of M1-92, perhaps indicating that the outflow is the result of a precessing or wobbling jet.

Binary star evolution is a popular scheme for producing bipolar morphology in PNe (e.g. Livio \& Soker 1988). In addition, an accretion disk in a binary system could account for the collimated outflow seen in M1-92. Alternatively, Frank, Balick, \& Livio (this volume) propose a scenario to produce jets in PNe that is a natural outcome of the interacting 


\section{POSTERS}
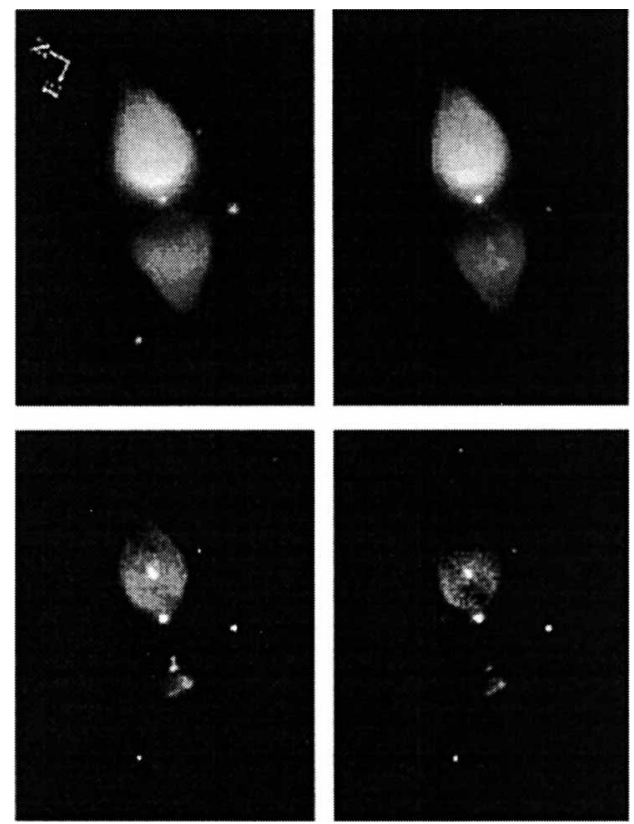

Figure 1: WFPC2 images of M1-92. The gray scale images shown are log(intensity) plots. The images are 13.65 " $x$ 18.2" in extent. Upper-left. The continuum band image of M192. The overall distribution of the continuum emission is smooth. Upper-right. The continuum subtracted $\mathrm{H} \alpha$ image. The morphology is similar to that seen in the continuum band. Lower-left. The continuum subtracted [O I] image. The emission is concentrated in a bright spot in the NW lobe and a series of clumps in the SE lobe. This emission is produced via shock heating and results as a collimated outflow impacts the bipolar lobes. Lower-right. The continuum subtracted [S II] image. The morphology is similar to that seen in [O I], indicating that the [S II] emission is also formed in the collimated outflow.

winds model. The possible origin of the jet is discussed in detail in Trammell \& Goodrich (1996).

\section{REFERENCES}

Frank, A., Balick, B., \& Livio, M. 1997, this volume.

Livio, M., \& Soker, N. 1988, ApJ, 329, 764.

Solf, J. 1994, A\&A, 282, 567.

Trammell, S. R., Dinerstein, H. L., \& Goodrich, R. W. 1993, ApJ, 402, 249.

Trammell, S. R. \& Goodrich, R. W. 1996, ApJ, 468, L107.

Support for this research was provided in part by NASA through a grant from the Space Telescope Science Institute. Support for the ground-based observations was provided by NSF grant 91-15101. S.R.T. was supported as a Grainger Postdoctoral Research Fellow. 